Supporting Information for the article

\title{
Measurements of Static and Dynamic Bubble Surface Tension Using a Deformation-Based Microfluidic Tensiometer
}

Shihao Liu', Cari S.Dutcher²,*(cdutcher@umn.edu)

${ }^{1}$ Department of Mechanical Engineering, University of Minnesota, Twin Cities, Minneapolis, Minnesota 55455, USA

${ }^{2}$ Department of Mechanical Engineering, Department of Chemical Engineering and Materials Science, University of Minnesota, Twin Cities, Minneapolis, Minnesota 55455, USA

\section{Microfluidic surface tension measurement}

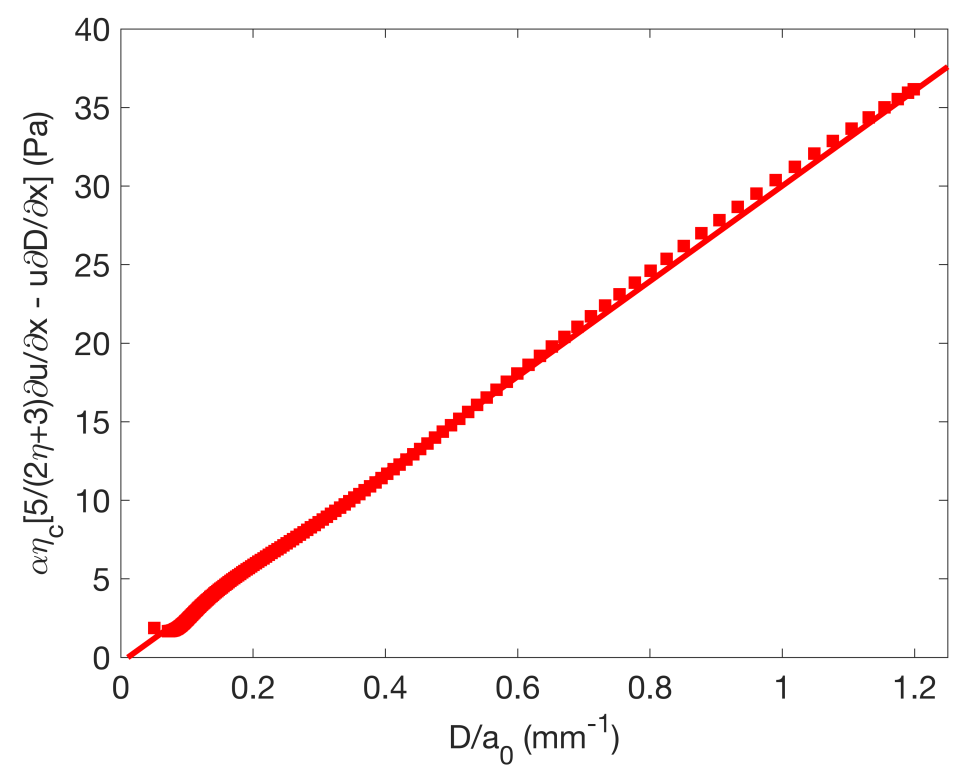

Figure S1-1 Surface tension plot for 100\% 1,2-pentanediol. The plot is based on equation (1) in the manuscript. The slope provides surface tension value of the chosen bubble to be 30.34 $\mathrm{mN} / \mathrm{m}$. Pendant drop measurement gives $29.97 \pm 0.66 \mathrm{mN} / \mathrm{m}$. 


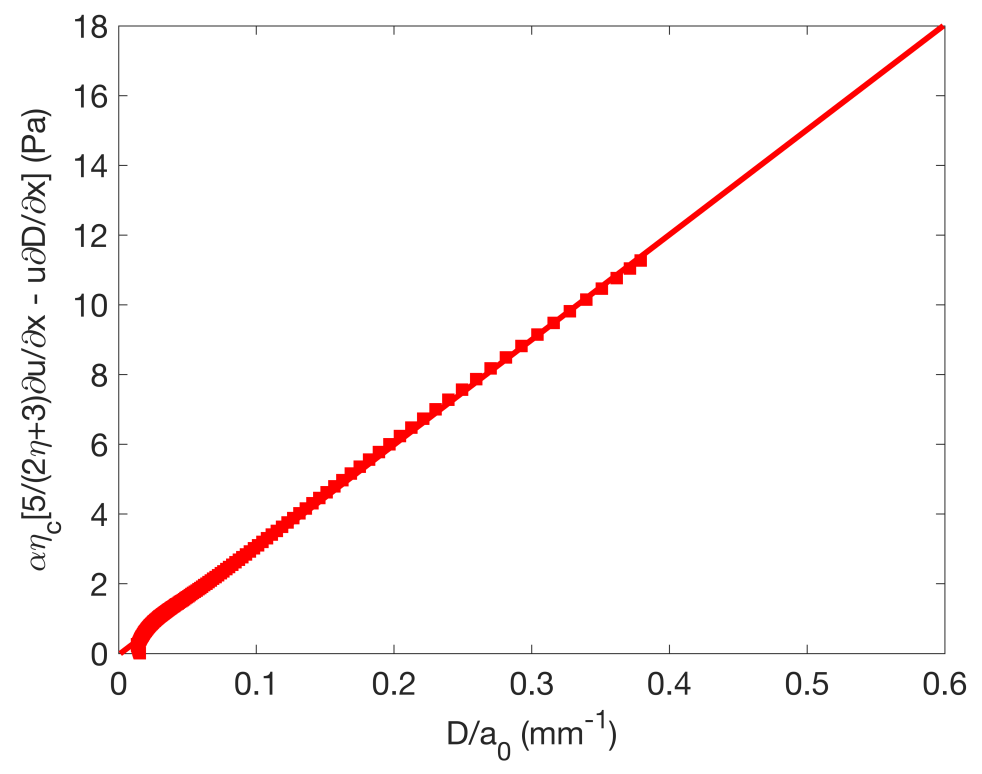

Figure S1-2 Surface tension plot for 75\% 1,2-pentanediol. The plot is based on equation (1) in the manuscript. The slope provides surface tension value of the chosen bubble to be 30.26 $\mathrm{mN} / \mathrm{m}$. Pendant drop measurement gives $30.58 \pm 0.19 \mathrm{mN} / \mathrm{m}$.

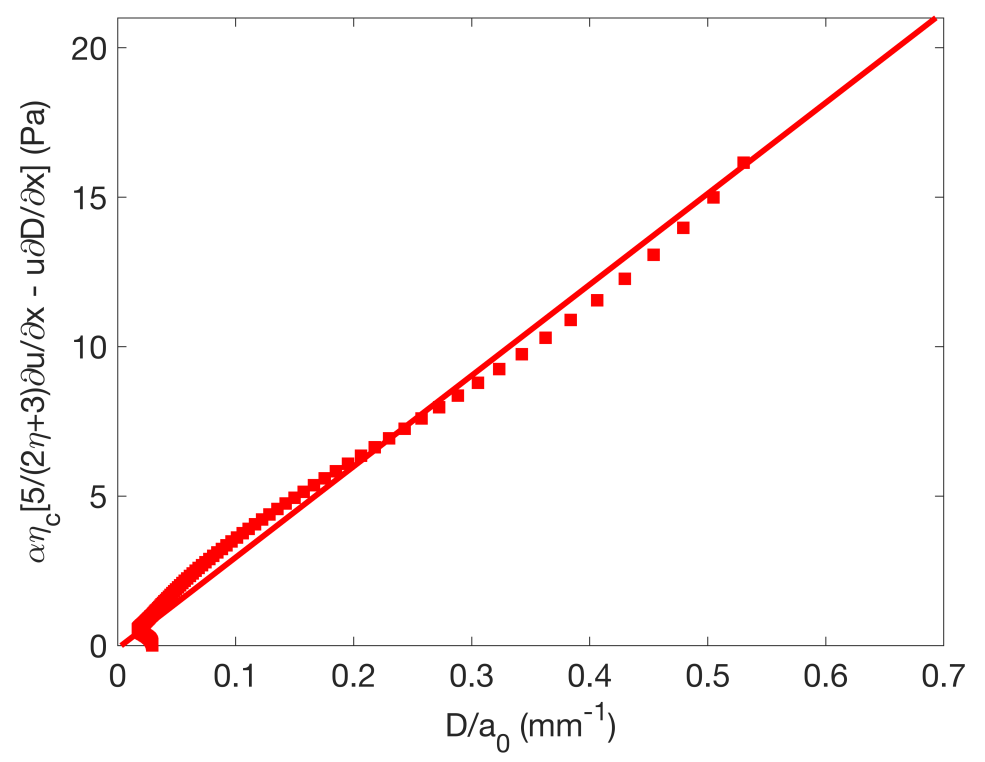

Figure S1-3 Surface tension plot for 75\% 1,2-hexanediol. The plot is based on equation (1) in the manuscript. The slope provides surface tension value of the chosen bubble to be 30.43 $\mathrm{mN} / \mathrm{m}$. Pendant drop measurement gives $30.19 \pm 1.11 \mathrm{mN} / \mathrm{m}$. 


\section{Bubble z-location with time}

To determine how long it takes for a bubble generated in microfluidic channel move up to the upper channel wall, a force balance-based description is used as below.

$$
\rho_{d} V \frac{d w}{d t}=\rho_{c} g V-6 \pi \eta_{c} a_{0} w-\rho_{d} g V
$$

where $\rho$ denotes the fluid density, $V$ represents bubble volume, $a_{0}$ is the bubble radius, $\eta$ the dynamic viscosity, $w$ is the velocity of the bubble in the direction perpendicular to the flow (we call it z-direction below) and $g$ is the gravitational acceleration. The subscript ' $c$ ' and ' $d$ ' represent continuous phase (surrounding liquid) and dispersed phase (air) in the experiments.

Plugging $\rho_{c}=1000 \mathrm{~kg} / \mathrm{m}^{3}, \rho_{d}=1.2 \mathrm{~kg} / \mathrm{m}^{3}, a_{0}=50 \times 10^{-6} \mathrm{~m}, \eta_{c}=40 \times 10^{-3} \mathrm{~Pa} \cdot \mathrm{s}$ and $g=10 \mathrm{~N} / \mathrm{kg}$ into equation (1) gives

$$
\frac{d w}{d t}=8316.67-6 \times 10^{7} w
$$

Let $A=8316.67, B=6 \times 10^{7}$, then

$$
w=A / B \times\left(1-\exp ^{-B t}\right)
$$

The translation in z-direction $H$ can be found by integrating equation (3), which gives

$$
H=A / B \times t-A / B^{2} \times\left(1-\exp ^{-B t}\right)
$$

The result indicates the term $A / B \times t$ dominates the other because (1) $A / B^{2}$ is always small and (2) $\exp ^{-B t}$ is always close to zero due to the large value of $B$. Given bubble diameter is 100 micrometers and the channel height is 125 micrometers, the result shows the bubble will arrive at the upper wall in less than 100 milliseconds, half of the typical time from generation to the first tensiometer (see Figure 6 caption). Because $B$ is proportional $\eta_{c}$, bubbles in the most viscous system $(100 \%$ 1,4-butanediol) in this work will take the longest time to reach the upper wall. For more accurate calculation, effects of non-uniform pressure distribution, the fluid surrounding the bubble that must be displaced, and the upper wall should also be 
considered.

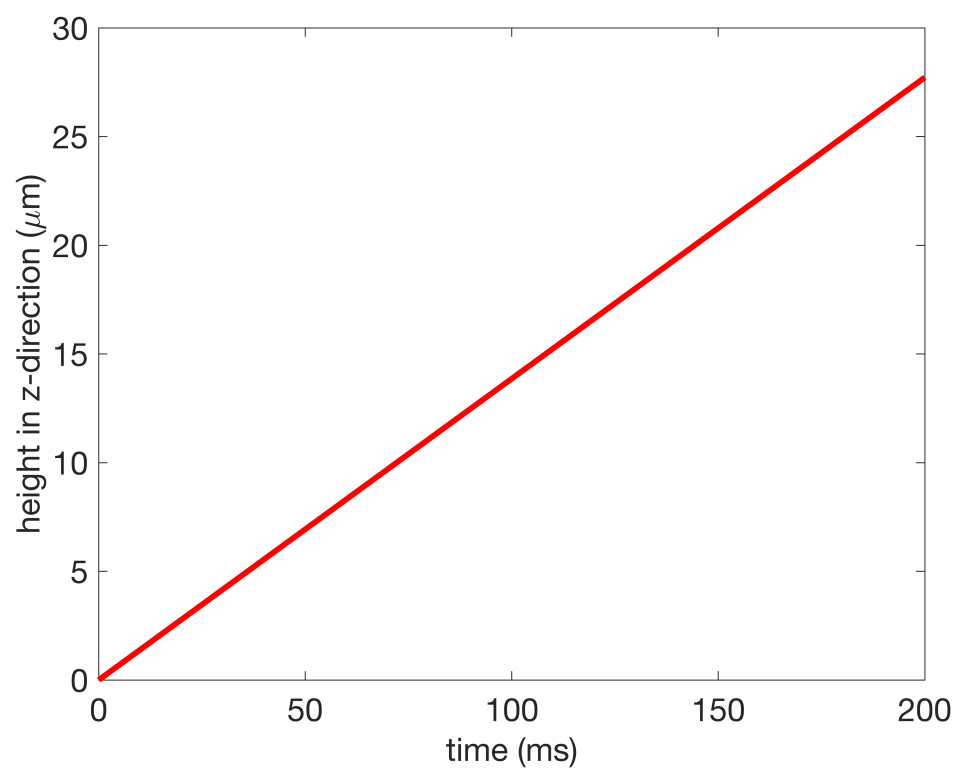

Figure S2 Plot showing the location of bubble in z-direction with time. Linear term in equation (4) dominates over the exponential term.

\section{Fitting pendant drop data of 500 ppm Dow $502 \mathrm{~W}$ in $75 \%$ 1,4-butanediol}

- $\Delta t$ is an adjustable parameter from $11 \mathrm{~s}$ to $20 \mathrm{~s}$

Table S1. Summary of adjusted and fitted parameters using equation (8) in the manuscript for the pendant drop dynamic surface tension measurement of $500 \mathrm{ppm}$ Dow 502W in 75\% 1,4-butanediol

\begin{tabular}{ccccc}
\hline$\Delta t(\mathrm{~s}$, adjust $)$ & $\gamma_{\mathrm{m}}(\mathrm{mN} / \mathrm{m}, \mathrm{fit})$ & $\alpha(\mathrm{fit})$ & $t^{*}(\mathrm{~s}, \mathrm{fit})$ & $\mathrm{RMSE}(\mathrm{mN} / \mathrm{m})$ \\
\hline 11 & 24.8791 & 0.5190 & 287.9154 & 0.2837 \\
12 & 26.0202 & 0.5419 & 241.3690 & 0.2822 \\
13 & 26.9555 & 0.5643 & 208.8814 & 0.2809 \\
14 & 27.7369 & 0.5864 & 185.2942 & 0.2797 \\
15 & 28.4001 & 0.6081 & 167.6274 & 0.2787 \\
16 & 28.9706 & 0.6295 & 154.0601 & 0.2777 \\
17 & 29.4669 & 0.6506 & 143.4264 & 0.2769 \\
18 & 29.9027 & 0.6714 & 134.9594 & 0.2761 \\
19 & 30.2892 & 0.6920 & 128.1122 & 0.2754 \\
20 & 30.6340 & 0.7123 & 122.5255 & 0.2748 \\
\hline
\end{tabular}


- $\Delta t$ is fitted with $t^{*}, \alpha$ and $\gamma_{\mathrm{m}}$ without the requirement about the range of $\Delta t$

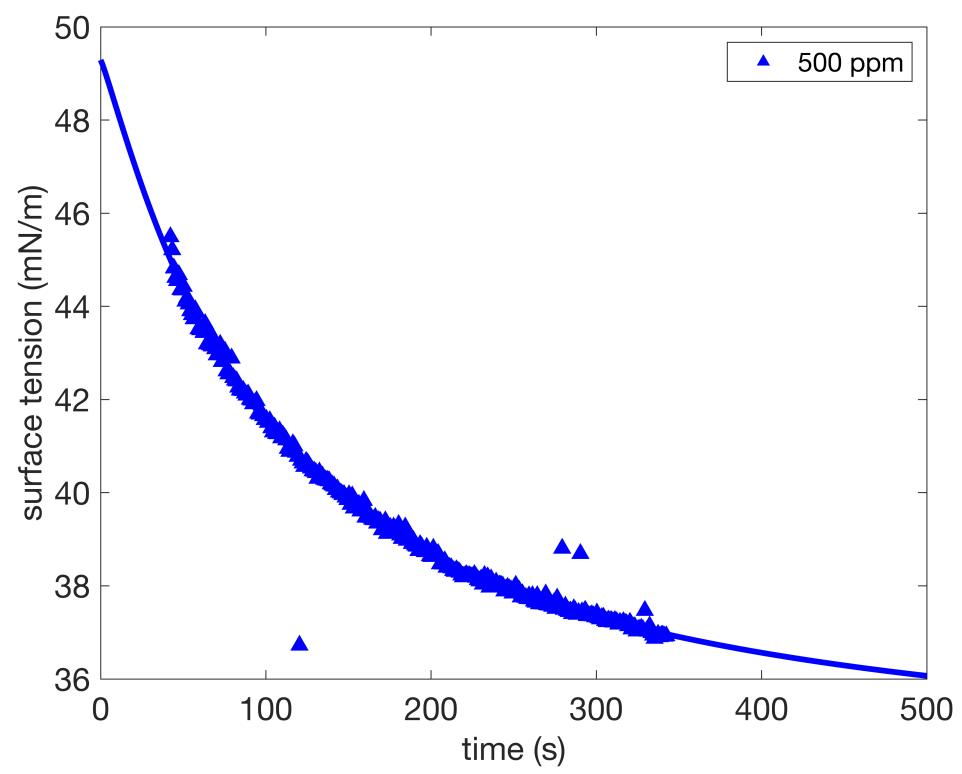

Figure S3. Dynamic surface tension measurement for $500 \mathrm{ppm}$ Dow $502 \mathrm{~W}$ dissolved in $75 \%$ 1,4-butanediol aqueous solutions using pendant drop. The solid line is plotted from $0 \mathrm{~s}$ to 10000 $\mathrm{s}$ based on equation (8) in the manuscript with four fitted parameters $\Delta t=42.29 \mathrm{~s}, \gamma_{\mathrm{m}}=33.98$ $\mathrm{mN} / \mathrm{m}, \alpha=1.13$ and $t^{*}=97.41 .95 \%$ confidence interval for the four fitted parameters are [23.63, 60.95], [32.58, 35.39], [0.79, 1.47], and [89.57, 105.26].

\section{Dimensions of microfluidic channel used for surface tension measurement}

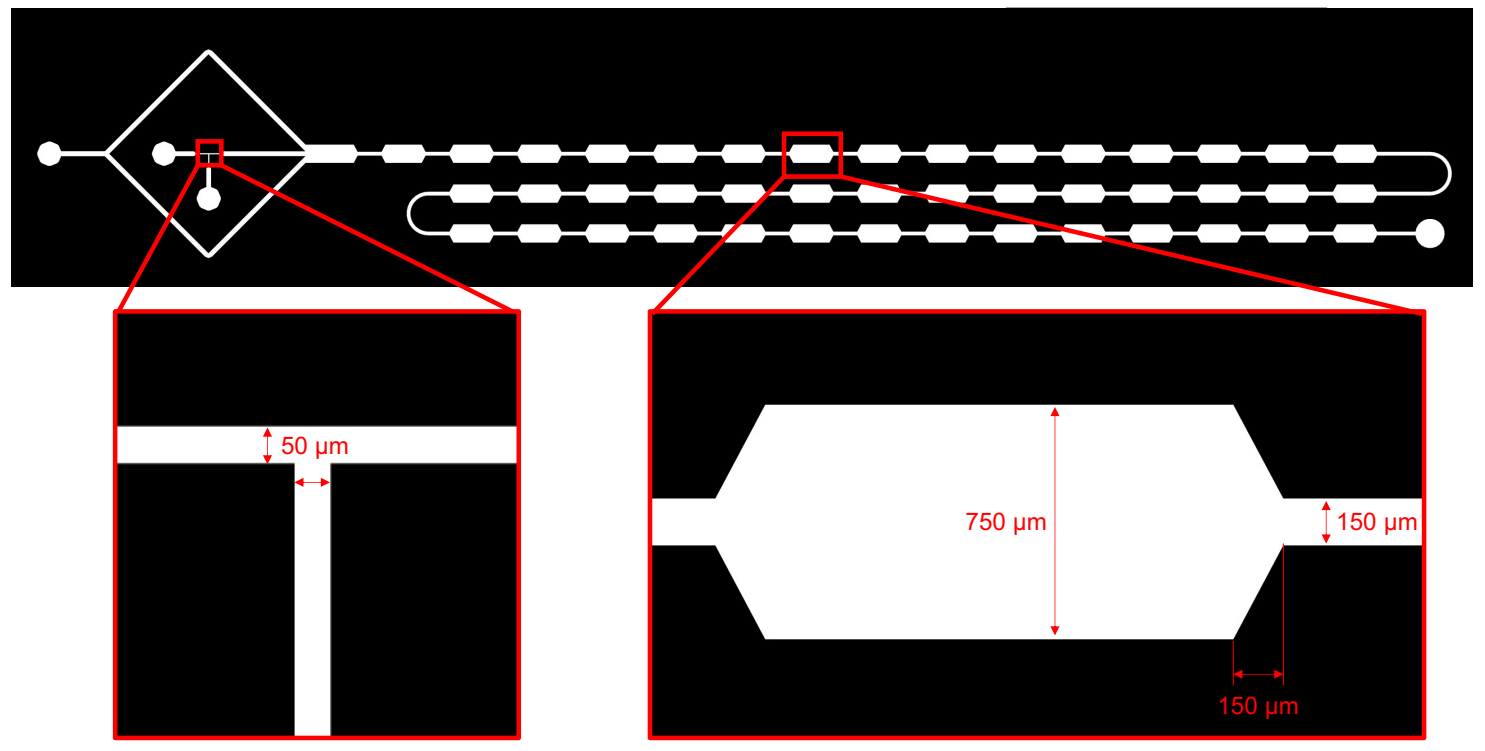

Figure S4. Schematic of microfluidic channel used for surface tension measurement. Channel height is 125 micrometers. 


\section{Relations of surface tension with dimensional variables}

- $100 \%$ 1,4-butanediol

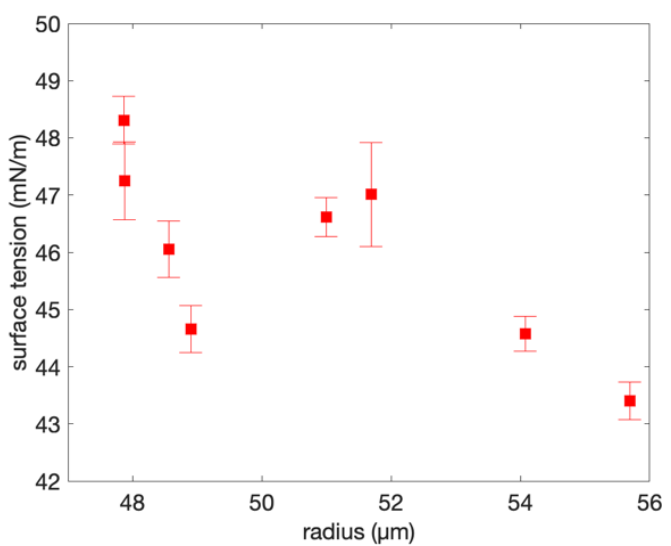

(a)

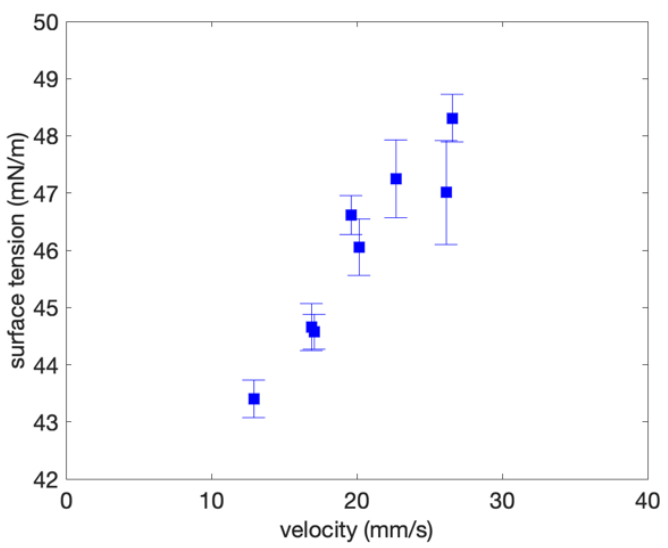

(b)

Figure S5-1. Relations of surface tension measured for 100\% 1,4-butanediol with (a) bubble radius and (b) bubble velocity.

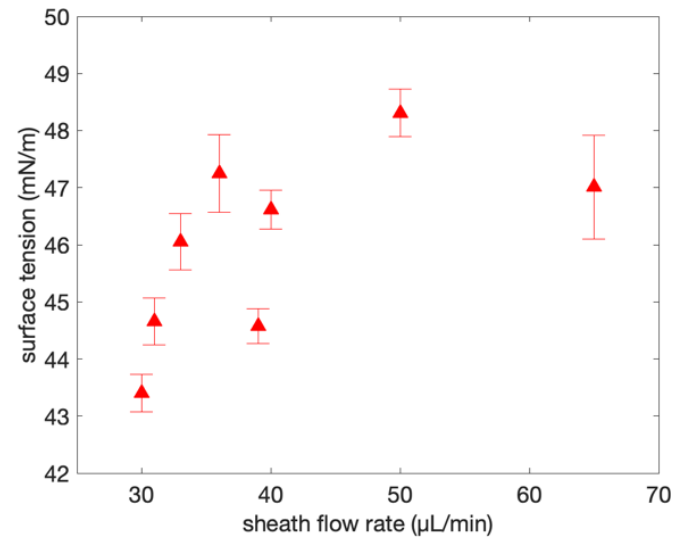

(a)

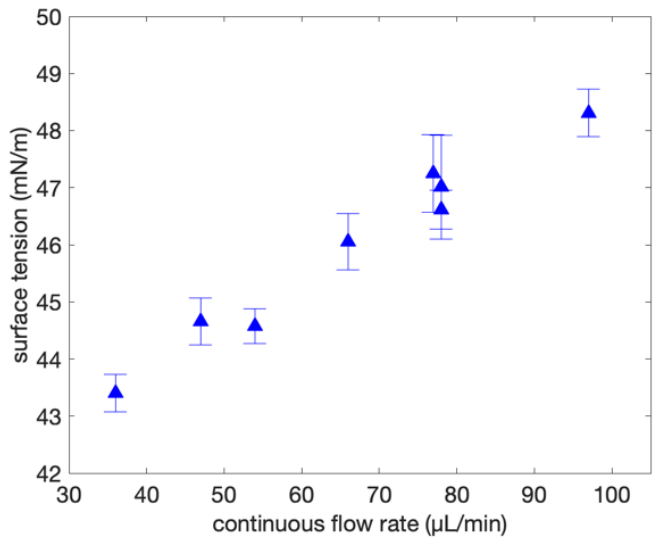

(b)

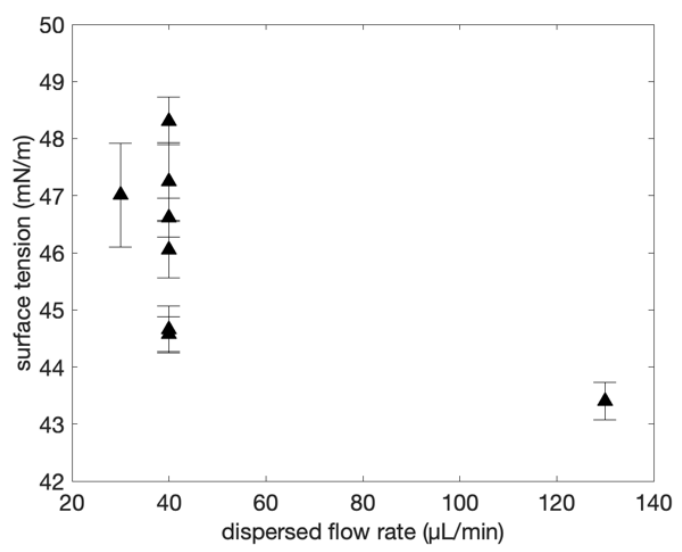

(c)

Figure S5-2. Relations of surface tension measured for 100\% 1,4-butanediol with flow rates of (a) sheath phase, (b) continuous phase and (c) dispersed phase. 
- $100 \%$ 1,2-pentanediol

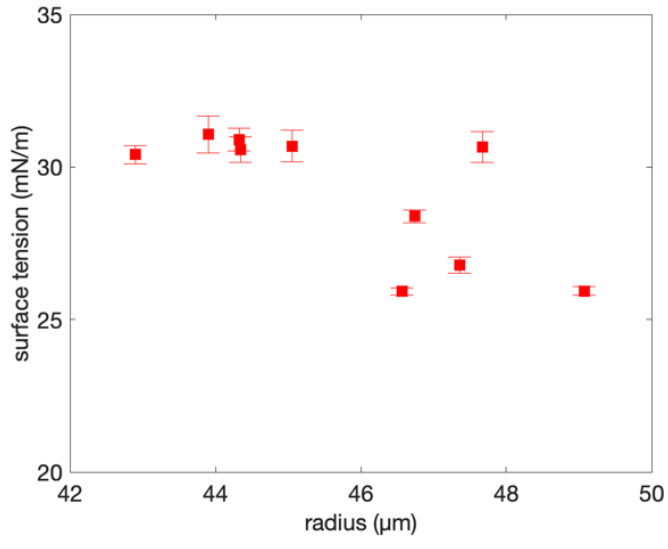

(a)

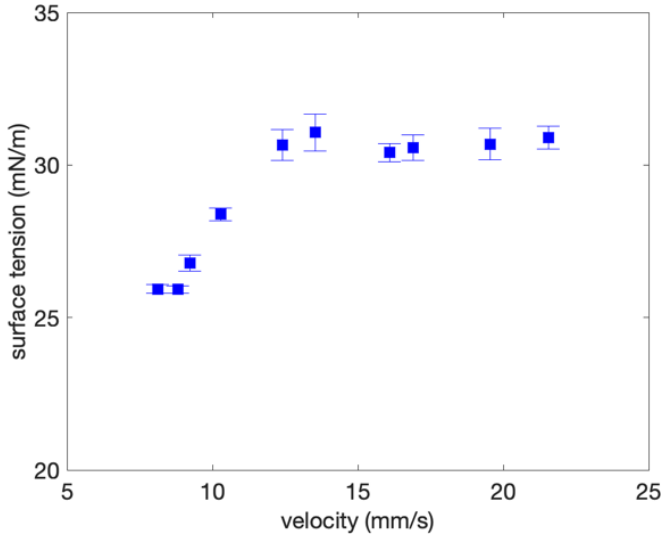

(b)

Figure S5-3. Relations of surface tension measured for 100\% 1,2-pentanediol with (a) bubble radius and (b) bubble velocity.

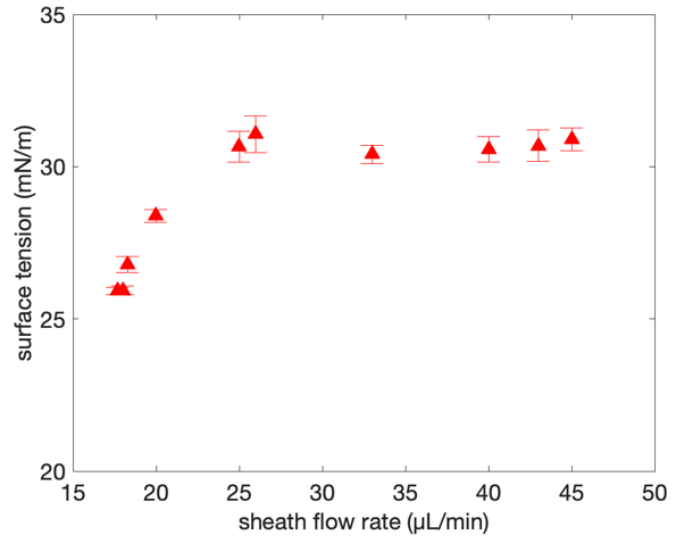

(a)

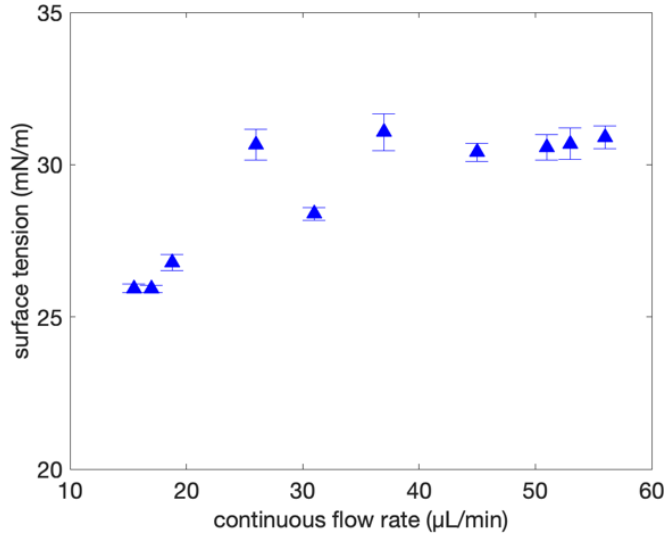

(b)

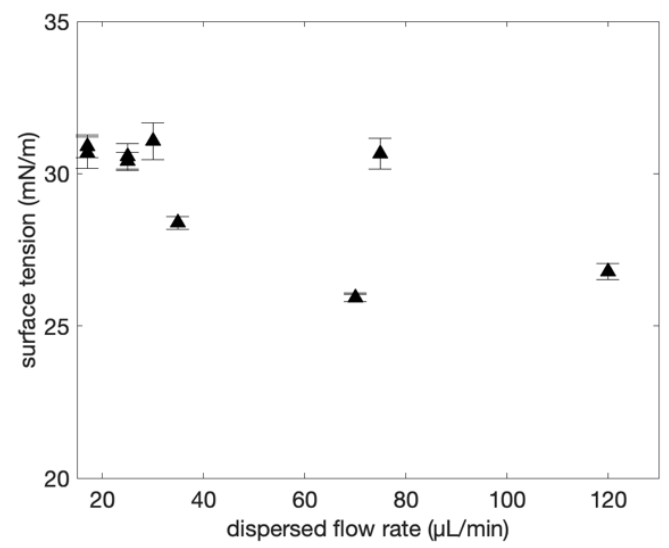

(c)

Figure S5-4. Relations of surface tension measured for 100\% 1,2-pentanediol with flow rates of (a) sheath phase, (b) continuous phase and (c) dispersed phase. 
- $100 \%$ 1,2-hexanediol

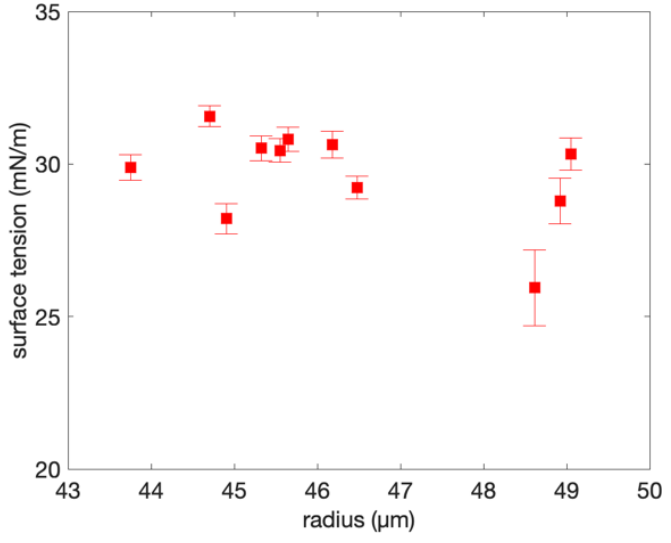

(a)

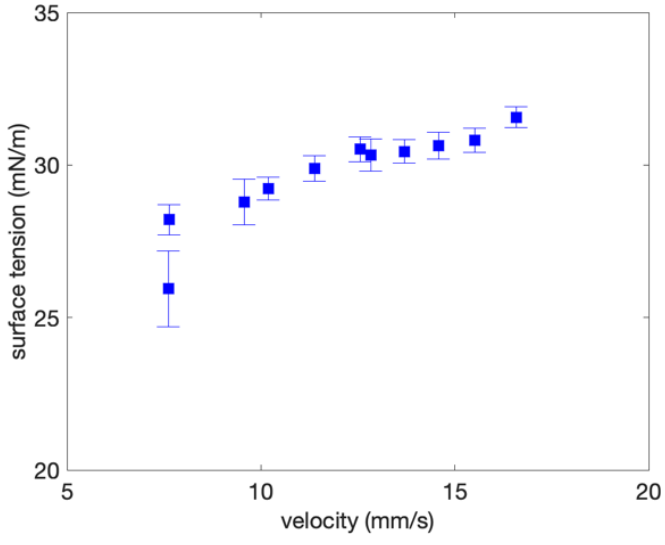

(b)

Figure S5-5. Relations of surface tension measured for 100\% 1,2-hexanediol with (a) bubble radius and (b) bubble velocity.

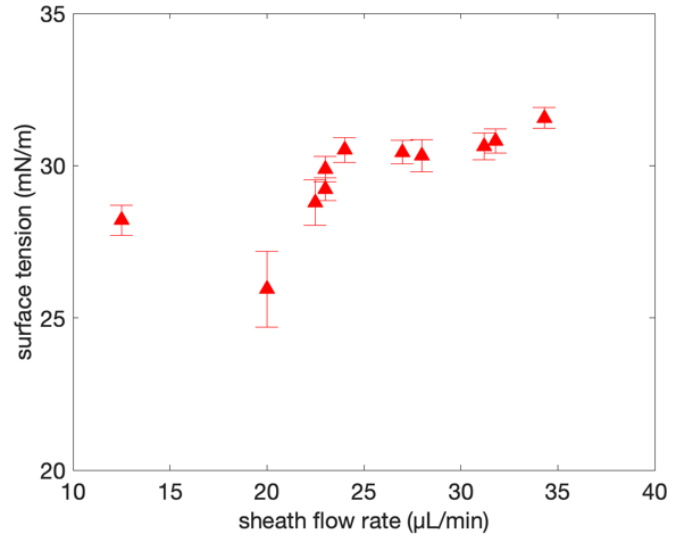

(a)

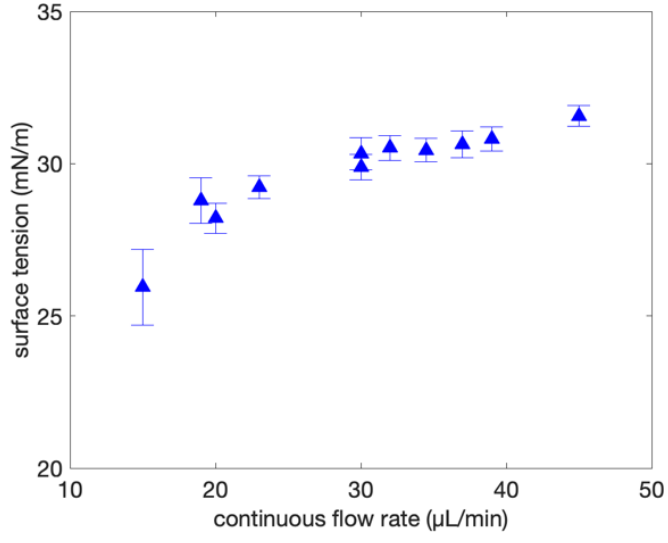

(b)

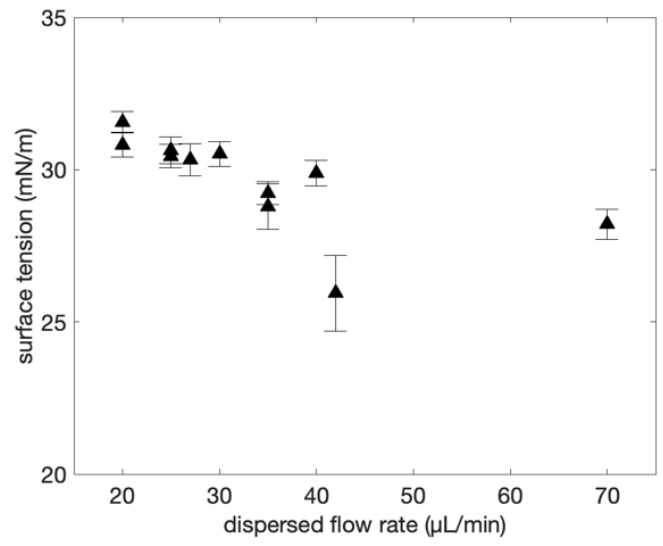

(c)

Figure S5-6. Relations of surface tension measured for 100\% 1,2-hexanediol with flow rates of (a) sheath phase, (b) continuous phase and (c) dispersed phase. 
- $75 \%$ 1,4-butanediol

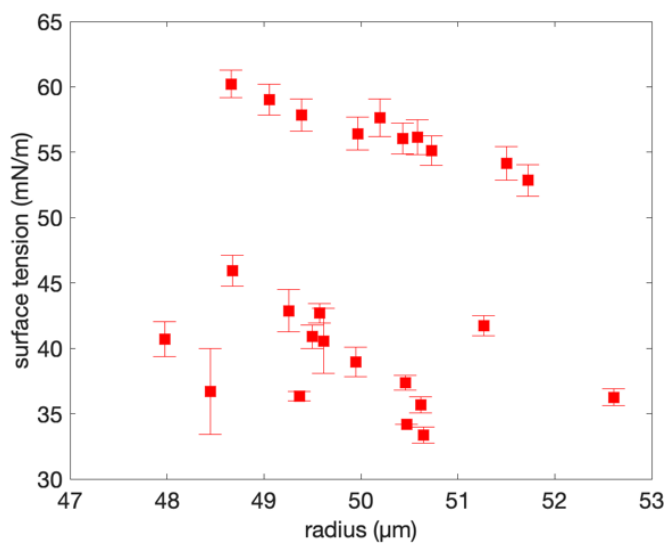

(a)

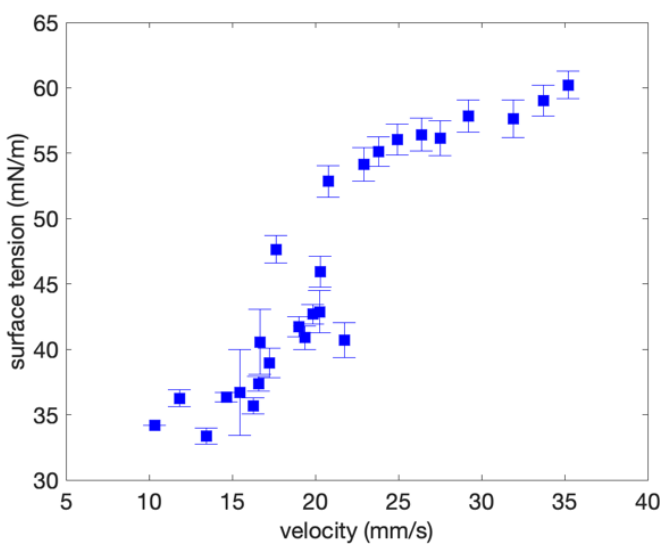

(b)

Figure S5-7. Relations of surface tension measured for 75\% 1,4-butanediol with (a) bubble radius and (b) bubble velocity.

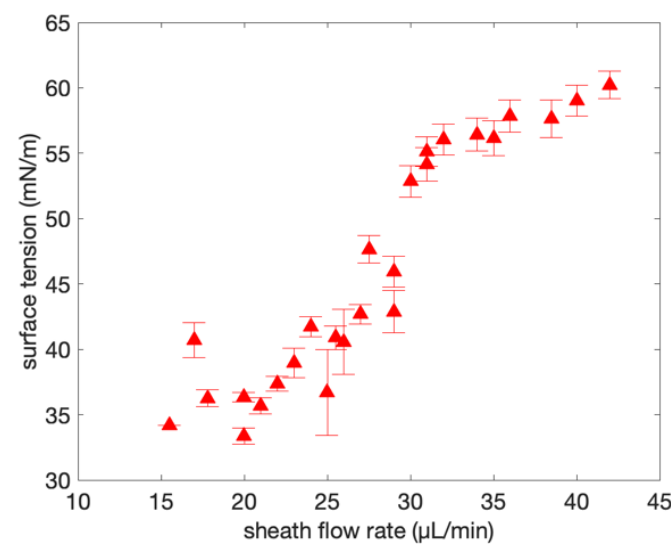

(a)

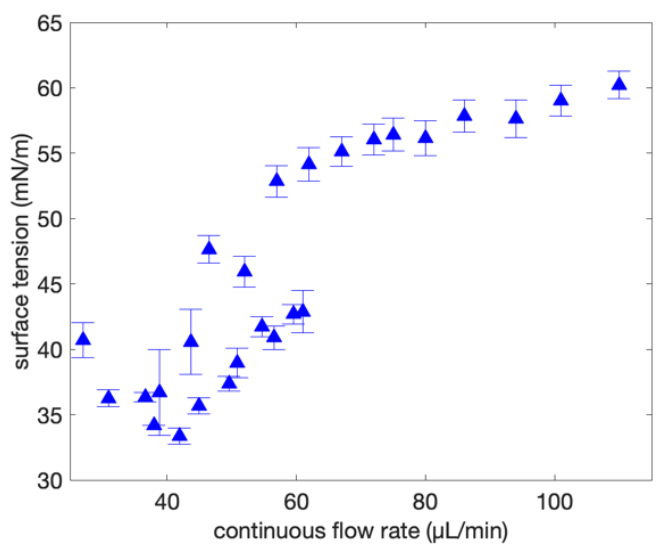

(b)

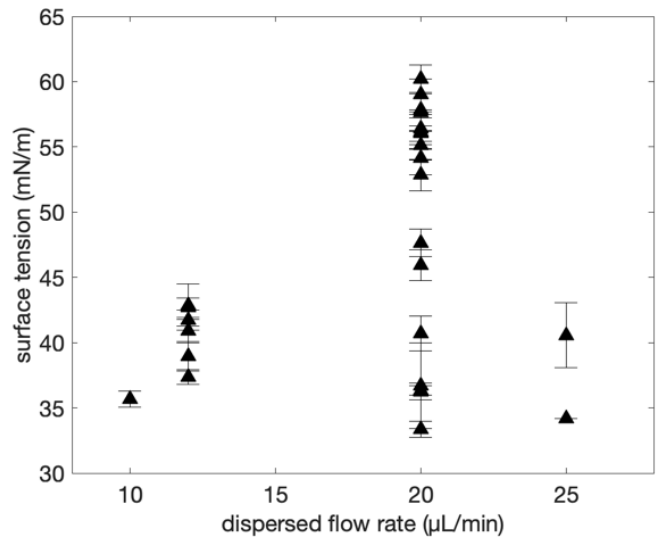

(c)

Figure S5-8. Relations of surface tension measured for 75\% 1,4-butanediol with flow rates of (a) sheath phase, (b) continuous phase and (c) dispersed phase 
- $75 \%$ 1,2-pentanediol

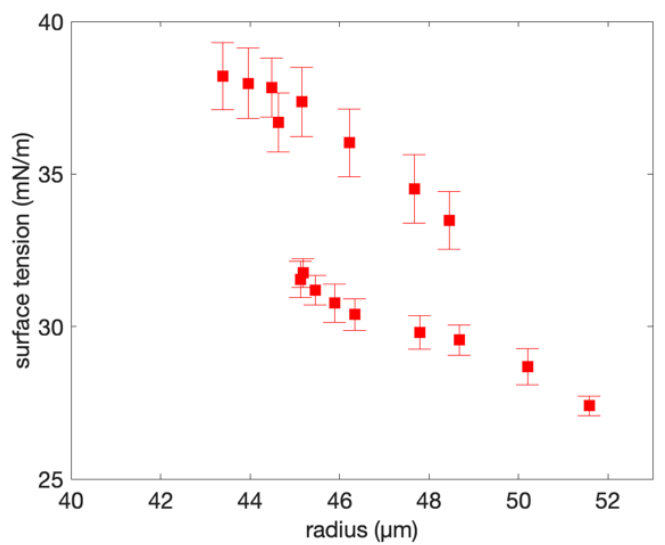

(a)

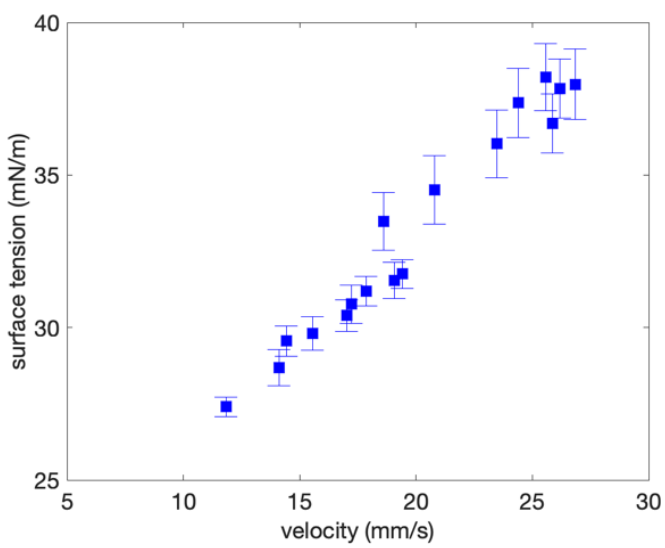

(b)

Figure S5-9. Relations of surface tension measured for 75\% 1,2-pentanediol with (a) bubble radius and (b) bubble velocity.

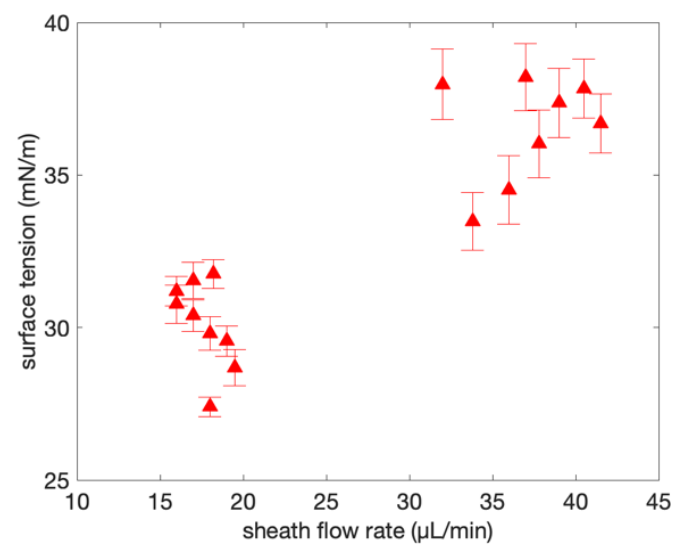

(a)

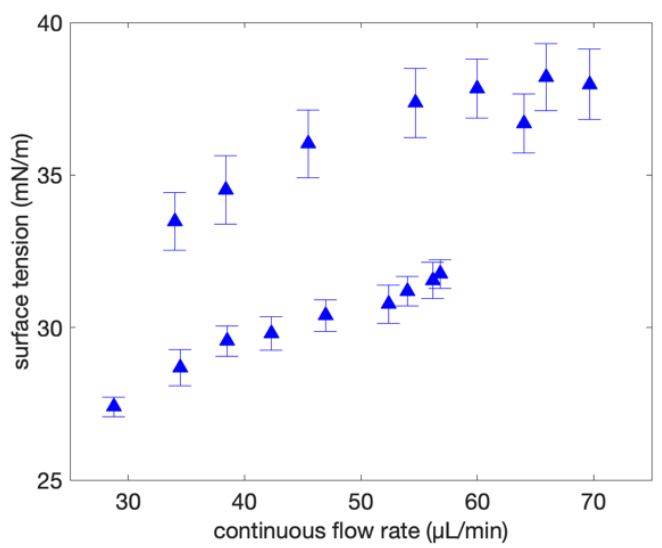

(b)

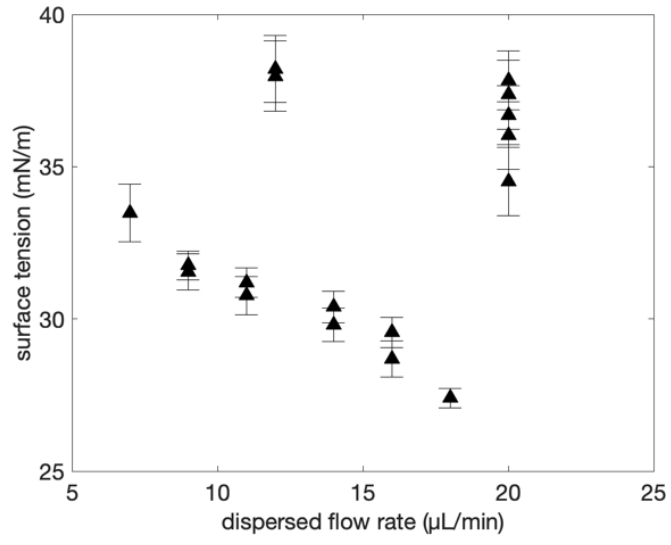

(c)

Figure S5-10. Relations of surface tension measured for 75\% 1,2-pentanediol with flow rates of (a) sheath phase, (b) continuous phase and (c) dispersed phase. 
- $75 \%$ 1,2-hexanediol

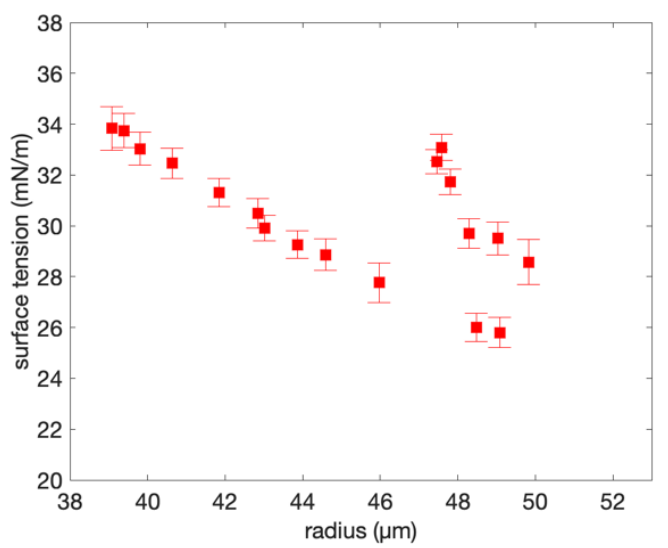

(a)

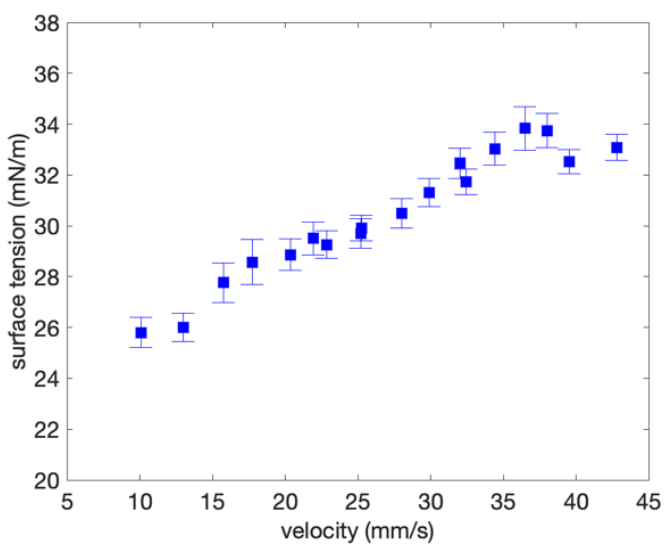

(b)

Figure S5-11. Relations of surface tension measured for 75\% 1,2-hexanediol with (a) bubble radius and (b) bubble velocity.

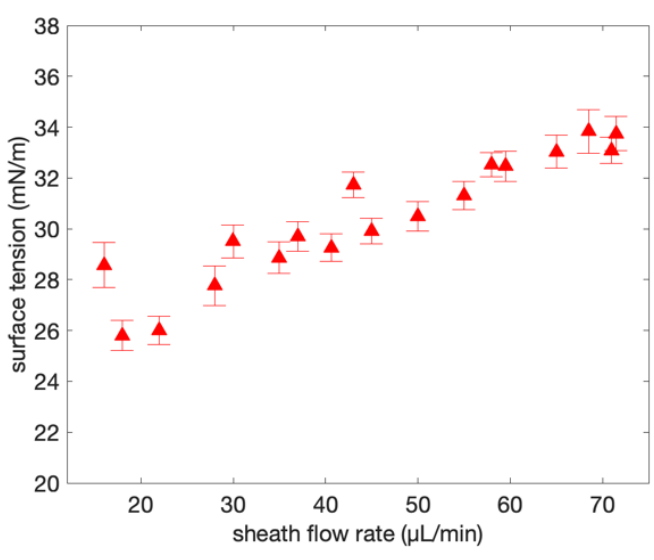

(a)

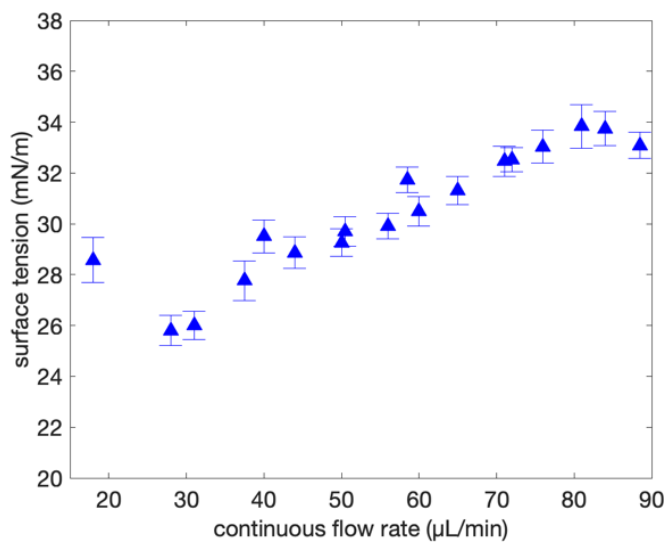

(b)

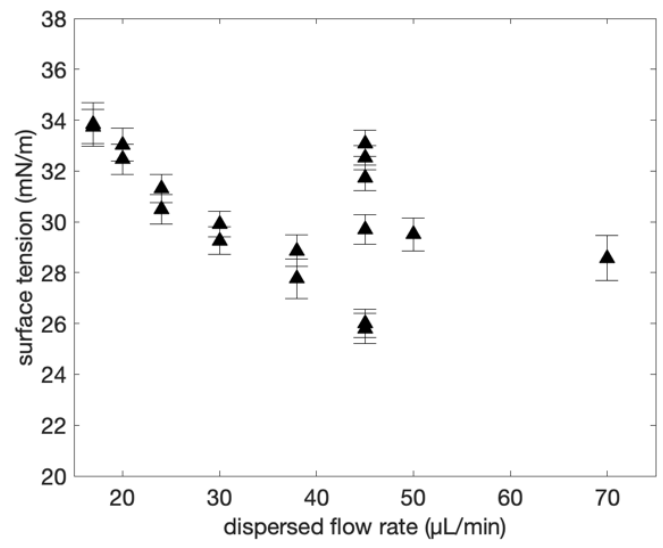

(c)

Figure S5-12. Relations of surface tension measured for 75\% 1,2-hexanediol with flow rates of (a) sheath phase, (b) continuous phase and (c) dispersed phase. 


\section{Range of flow rates for different systems}

Table S2. Flow rate range for different systems and different ports shown in Figure 1c in the manuscript (Sheath flow: S; Continuous flow: C; Dispersed flow: D)

\begin{tabular}{cccc}
\hline Systems & $\mathbf{S}(\boldsymbol{\mu L} / \mathbf{m i n})$ & $\mathbf{C}(\boldsymbol{\mu L} / \mathbf{m i n})$ & $\mathbf{D}(\boldsymbol{\mu L} / \mathbf{m i n})$ \\
\hline $100 \%$ 1,4-butanediol & $30.0-65.0$ & $36.0-97.0$ & $30.0-130.0$ \\
100\% 1,2-pentanediol & $17.7-45.0$ & $15.5-56.0$ & $17.0-120.0$ \\
100\% 1,2-hexanediol & $12.5-34.3$ & $15.0-45.0$ & $20.0-70.0$ \\
$75 \%$ 1,4-butanediol & $15.5-42.0$ & $27.0-110.0$ & $10.0-25.0$ \\
75\% 1,2-pentanediol & $16.0-41.5$ & $28.8-69.7$ & $7.0-20.0$ \\
$75 \%$ 1,2-hexanediol & $16.0-71.5$ & $18.0-88.5$ & $17.0-70.0$ \\
$75 \%$ 1,4-butanediol + 50 ppm Dow & $34.0-46.0$ & $30.0-44.0$ & 20.0 \\
$75 \%$ 1,4-butanediol + 500 ppm Dow & $54.0-61.0$ & $38.5-50.0$ & 20.0 \\
\hline
\end{tabular}

